\title{
Estimation of the tangential transverse vibrations effect on the friction force with the use of LuGre model
}

Received: 29 July 2019 / Revised: 31 March 2021 / Accepted: 14 June 2021 / Published online: 17 July 2021

(C) The Author(s) 2021

\begin{abstract}
In this work, a novel dynamic computational model developed for the purpose of modelling the phenomenon of friction force reduction in a sliding motion at tangential transverse vibrations of elastic support is presented. In contrast to the currently used so-called kinematic models, this model takes into account the mass of a sliding and simultaneously subjected to vibrations body. It is based on the dynamic equations of motion of this body. For the description of the friction force, the LuGre model was used which considers both the compliance of the contact zone and its damping. Considered in this model are also compliance and damping of the drive. The model assumes that vibrations are not imposed directly on the shifting body but are transferred to it from the vibrating elastic support. The developed model has been implemented in the Matlab/Simulink environment and subsequently utilised in simulating analyses. The results of these were compared with the results of experiments which were carried out using a designated, in-house built, experimental rig. An excellent compliance has been achieved.
\end{abstract}

\section{Introduction}

Until now, in simulating analyses of the influence of tangential transverse vibrations on the friction force in sliding motion so-called kinematic models have been mostly utilised [1-9], which do not consider the mass of a shifted body that simultaneously undergoes vibration motion. Moreover, for describing the friction force in these models a simple Coulomb friction model was predominantly used, which does not consider compliance of the contact zone between the shifted body and the support. Not considered was also the compliance of a drive. These drawbacks caused significant discrepancies between the outcomes of simulating analyses and experiments due to which it was possible to talk about a rather qualitative consistency, and not the quantitative one.

In the previous paper, published in 2015 [10], the authors presented a novel, based on dynamic equations of motion of a shifted body, computational model for analysing the influence of tangential transverse vibrations on the frictional resistance in sliding motion. According to that model, they achieved very good compliance of simulation analyses results with those of experiments. In that model, for the description of friction force, Dahl's [11] model was used.

Now, the model originally presented in paper [10] has been significantly improved by the use of the socalled LuGre model for friction force description [12, 13], which considers both the compliance of the contact zone and its damping. Considered in this model are also compliance and damping of the drive. The developed

P. Gutowski $(\bowtie) \cdot$ M. Leus

Faculty of Mechanical Engineering and Mechatronics, West Pomeranian University of Technology Szczecin, Al. Piastów 19, 70-310 Szczecin, Poland

e-mail: pawel.gutowski@zut.edu.pl

M. Leus

e-mail: mariusz.leus@zut.edu.pl 

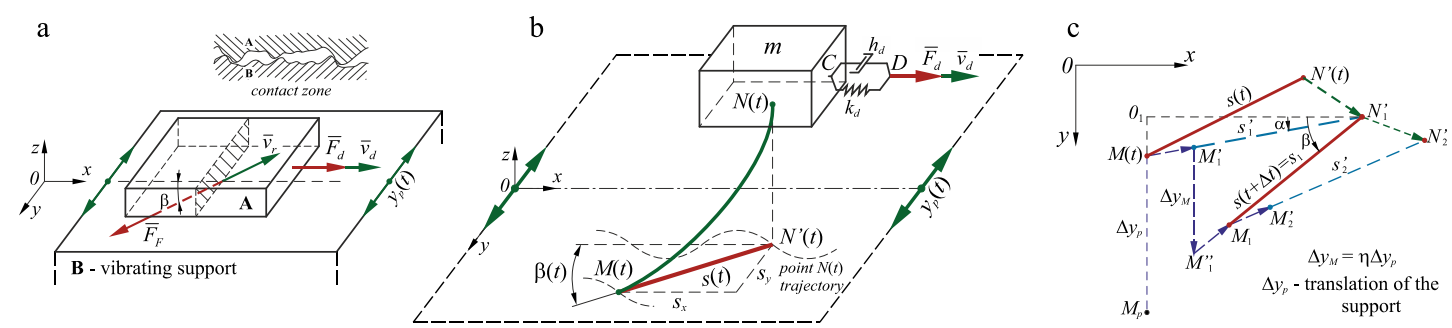

Fig. 1 Modelling of roughness asperities elastic deformation in the contact zone at transverse vibrations; a frictional pair, $\mathbf{b}$ elastic deformation $s(t)$ of contact in the plane of sliding, $\mathbf{c}$ changes of $s(t)$ in consecutive phases of sliding motion

model has been utilised in simulating analyses of the influence of tangential transverse vibrations on the friction force. For its verification, experiments were carried out using a designated, in-house built, experimental rig described in detail in $[10,14]$. Very good compliance of the simulation analyses and experimental tests results has been achieved.

\section{Description of the model}

In the physical model (Fig. 1), it was assumed that the upper body $(A)$ with the mass $m$ is being shifted by a drive with rigidity $k_{\mathrm{d}}$ and damping $h_{\mathrm{d}}$ over the support $(B)$ which can, at any optional instant, be put into vibrating motion in direction tangential, perpendicular to the shift. The direction of driving force $F_{\mathrm{d}}$ necessary for initiating the body movement in sliding motion and maintaining this motion is compatible with the direction of macroscopic sliding. It has been assumed that the contact zone, formed by roughness protrusions of the shifted body and support remaining in mutual contact, is a deformable zone which under tangential vibrations can be modelled by a single artificial rod-like, nonlinear elasto-damping element $M N$ (Fig. 1b) which models the contact's rigidity and damping in the tangential direction.

An elastic deformation of this zone can be presented in the model as a result of mutual movement of the end points $M$ and $N$ of this element. In the plane of contact, its measure is a distance $s$ between points $M$ and $N^{\prime}$, where $N^{\prime}$ is the projection of point $N$ on a plane of slide (Fig. 1b), and hence:

$$
s(t)=\sqrt{\left[N_{x}^{\prime}(t)-M_{x}(t)\right]^{2}+\left[M_{y}(t)-N_{y}^{\prime}(t)\right]^{2}} .
$$

The direction of this deformation is variable in time and oscillates around the direction of the macroscopic motion of the shifted body. At any instant $t$, it is determined by an angle $\beta$. This direction complies with that of relative motion and with the direction of relative velocity $\vec{v}_{\mathrm{r}}$ of the shifted body and vibrating support. The friction force vector $\vec{F}_{\mathrm{F}}$ (Fig. 1) is also placed along this direction.

In the LuGre model $[12,13]$, the magnitude of this force is determined by the relationship:

$$
F_{\mathrm{F}}=k_{\mathrm{t}} \cdot s+h_{\mathrm{t}} \cdot \dot{s}+h_{\mathrm{v}} \cdot v_{\mathrm{r}}
$$

where $s$-elastic deformation of the contact in the tangential direction, $k_{\mathrm{t}}$-coefficient of contact rigidity in the tangential direction, $h_{\mathrm{t}}$-contact damping coefficient at tangential deformation, $h_{\mathrm{v}}$-viscous damping coefficient, and $v_{\mathrm{r}}$-velocity of relative motion of the contact surfaces.

The rate $\dot{s}$ of contact elastic deformation, in this equation, is determined from the relationship:

$$
\dot{s}=\frac{\mathrm{d} s}{\mathrm{~d} t}=v_{\mathrm{r}}-\frac{\left|v_{\mathrm{r}}\right|}{g\left(v_{\mathrm{r}}\right)} \cdot s
$$

whilst:

$$
g\left(v_{\mathrm{r}}\right)=\frac{1}{k_{\mathrm{t}}} \cdot\left[F_{\mathrm{C}}+\left(F_{\mathrm{S}}-F_{\mathrm{C}}\right) \cdot e^{-\left(v_{\mathrm{r}} / v_{\mathrm{S}}\right)^{2}}\right]
$$

where $F_{\mathrm{C}}-$ Coulomb friction force, $F_{\mathrm{C}}=\mu \cdot F_{\mathrm{N}}, F_{\mathrm{S}}-$ static friction force, and $v_{\mathrm{S}}-$ Stribeck velocity. 
The value of the contact damping coefficient $h_{\mathrm{t}}$ at tangential deflection, in case of lack of sliding, is determined from relationship [15]:

$$
h_{\mathrm{t}}=2 \cdot \zeta \cdot \sqrt{m \cdot k_{\mathrm{t}}}
$$

In the case of sliding, this coefficient is a function of relative velocity $v_{\mathrm{r}}$ of surfaces in contact. It can be described [15] by the relationship:

$$
h_{\mathrm{t}}\left(v_{\mathrm{r}}\right)=2 \cdot \zeta \cdot \sqrt{m \cdot k_{\mathrm{t}}} \cdot e^{-\left(v_{\mathrm{r}} / v_{\mathrm{e}}\right)^{2}}
$$

where $m$-the mass of the shifted body, $\zeta$-coefficient of critical damping, and $v_{\mathrm{e}}=v_{\mathrm{s}}$.

From the LuGre model, it is easy to switch into the Dahl model-assuming that $F_{\mathrm{S}}=F_{\mathrm{C}}$ and that $h_{\mathrm{t}}=h_{\mathrm{v}}=0$. It is seen from relationships (2) and (3) that for determining the friction force $F_{\mathrm{F}}$, the elastic deformation $s$ of the contact in the plane of slide needs to be known. This deformation and its direction can be determined by analysing the motion of end points $M$ and $N$ of the $M N$ element. Their location at any moment of the shifted body motion is the effect of superposition of the translation caused by the driving force $\vec{F}_{\mathrm{d}}$ with that caused by support vibrations $y_{p}$.

At succeeding instances of motion, these end points change their relative location. That means that the elastic deformation $s$ of the contact, measured in the plane of sliding, also changes, in both magnitude and direction (Fig. 1c). In the developed procedure, these changes at each instant $\Delta t$ were separated into two phases. In the first of them, they are considered as a result of the motion of point $N$ rigidly connected with the shifted body, and in the second one as a result of the motion of an unbounded end $M$ of elastic element $M N$.

The motion of the body in the direction of axes $O \times$ and $O y$ is parallel to the plane of slide, and hence, the motion of points $N$ and $N^{\prime}$ in these directions can be described by the following equations:

$$
\begin{gathered}
m \cdot \ddot{x}=F_{\mathrm{d}}-F_{\mathrm{F}} \cdot \cos \beta, \\
m \cdot \ddot{y}=F_{\mathrm{F}} \cdot \sin \beta .
\end{gathered}
$$

The driving force $F_{\mathrm{d}}$ can be determined from a simple relationship:

$$
F_{\mathrm{d}}=\lambda_{\mathrm{d}} \cdot k_{\mathrm{d}}
$$

where $\lambda_{\mathrm{d}}$ - elastic deformation of the drive, and $k_{\mathrm{d}}$-coefficient of the drive rigidity.

The elastic deformation $\lambda_{\mathrm{d}}$ of the drive in the direction of macroscopic movement of the shifted body corresponds to the relative displacement of ends $C$ and $D$ of the elastic element $C D$ modelling this drive (Fig. 1b). Therefore, it can be determined from the dependence:

$$
\lambda_{\mathrm{d}}=\left(v_{\mathrm{d}} \cdot t-x\right)
$$

where $v_{\mathrm{d}}$-velocity of drive, $t$ - time, and $x$-displacement of a shifted body.

At an optional initial time instant $t$, i.e. at the beginning of a new time increment $\Delta t$ the end points $M$ and $N$, modelling the roughness protrusions have the coordinates $M_{x}(t)$ and $M_{y}(t)$ as well as $N_{x}(t)$ and $N_{y}(t)$, and hence $M(t)=\left[M_{x}(t), M_{y}(t)\right]$ and $N(t)=\left[N_{x}(t), N_{y}(t)\right]$. The point $N^{\prime}$, which is the projection of point $N$ on a plane of slide, has at this time the coordinates $N^{\prime}(t)=\left[N_{x}^{\prime}(t), N_{y}^{\prime}(t)\right]$, where $N_{x}^{\prime}(t)=N_{x}(t)$ and $N_{y}^{\prime}(t)=N_{y}(t)$.

At the first phase of motion, in each succeeding time interval $\Delta t$, point $N^{\prime}$ moves to the position $N_{1}^{\prime}$ assigned by the coordinates $N_{1 x}^{\prime}$ and $N_{1 y}^{\prime}$, where $N_{1 x}^{\prime}=N_{x}^{\prime}(t+\Delta t)=x$ and $N_{1 y}^{\prime}=N_{y}^{\prime}(t+\Delta t)=y$. The unconstrained end $M$ of the elastic element $M N$ moves, at the same time, along direction $M N_{1}^{\prime}$ to the new position designated as $M_{1}^{\prime}$. Thus an elastic deformation $s$ undergoes a change both in its direction—defined temporarily by angle $\alpha$, and in its magnitude.

The changes in magnitude of $s$ can be described by an increment $\Delta s$. Thus the instantaneous value of elastic deformation of element $M N$ at this phase of motion measured in the plane of sliding is:

$$
s_{1}^{\prime}=s+\Delta s \text {. }
$$

The value of increment $\Delta s$ can be determined with the use of Eq. (3). The following dependence is obtained:

$$
\Delta s=\left(v_{\mathrm{r} 1}-\frac{\left|v_{\mathrm{r} 1}\right|}{g\left(v_{\mathrm{r} 1}\right)} \cdot s\right) \cdot \Delta t .
$$


The relative velocity $v_{r 1}$ of roughness protrusions at this stage of movement can be calculated according to the relationship [10]:

$$
v_{\mathrm{r} 1} \approx \frac{M N_{1}^{\prime}-s}{\Delta t}
$$

where

$$
M N_{1}^{\prime}=\sqrt{\left[x-M_{x}(t)\right]^{2}+\left[M_{y}(t)-y\right]^{2}} .
$$

The direction of this velocity in relation to axis $x$ is determined by angle $\alpha$ (Fig. 1c).

After evaluation of the instantaneous value $s_{1}^{\prime}$ of elastic deformation of element $M N$, the location $M_{1}^{\prime}$ of the end point $M$ can be easily determined. From Fig. 1c, it is seen that coordinates of this point are as follows:

$$
\begin{gathered}
M_{1 x}^{\prime}=x-s_{1}^{\prime} \cdot \cos \alpha, \\
M_{1 y}^{\prime}=y+s_{1}^{\prime} \cdot \sin \alpha .
\end{gathered}
$$

The values of $\sin \alpha$ and $\cos \alpha$ are determined from the triangle $0_{1} N_{1}^{\prime} M(t)$. It is seen from this triangle that:

$$
\sin \alpha=0_{1} M / M N_{1}^{\prime} \text { and } \cos \alpha=0_{1} N_{1}^{\prime} / M N_{1}^{\prime}
$$

where

$$
0_{1} M=M_{y}(t)-y \text { and } 0_{1} N_{1}^{\prime}=x-M_{x}(t) .
$$

Simultaneously to the sliding motion of the upper body, the support over which this motion takes place undergoes vibrations, which are generated in transverse direction, according to an imposed equation, e.g.:

$$
y_{p}=u_{o} \cdot \sin (\omega t)
$$

where $u_{o}$-vibrations amplitude, $\omega$-rotational frequency, whilst $\omega=2 \cdot \pi \cdot f$, and $f$-vibrations frequency. The displacement of the support within time interval $\Delta t$ is:

$$
\Delta y_{p}=u_{o} \cdot[\sin \omega(t+\Delta t)-\sin (\omega t)] .
$$

In Fig. 1c this shift relates to the way $M M_{P}\left(\Delta y_{p}=M M_{P}\right)$. The motion of the support, however, is not entirely transferred to the sliding body. It is because of the fact that the end point $M$ of the elastic element $M N$ is not fixed to the support but slides over it. Hence, the displacement $y_{M}$ of this point along axis $y$ caused by vibrations comprises only a part of support displacement $y_{p}$ in this direction. Hence, it can be noted:

$$
y_{M}=\eta \cdot y_{p}
$$

where $\eta$ - coefficient of vibrations transfer.

The vibrations transfer coefficient $\eta$ from the support to an unbounded end $M$ of element $M N$ modeling the roughness protrusions of the shifting body is estimated as a consequence of tuning the friction force changes under the influence of vibrations obtained in simulation analyses, by means of numerical calculations, to the profiles determined experimentally. The tuning procedure is described in detail in our previous work [10].

Due to elastic deformation of element $M N$, its end point $M$ moves alongside direction $M N_{1}^{\prime}$. So at this phase of motion it is located at point $M_{1}^{\prime}$. Hence, the translocation of end point $M$ in the direction of axis $y$, caused by support vibrations, equals to $\Delta y_{M}=M_{1}^{\prime} M_{1}^{\prime \prime}=\eta \cdot M M_{P}$. As a result of this motion, there is a further change in the elastic deformation of element $M N$, from an intermediate value $s_{1}^{\prime}$, in the first phase of changes in time interval $\Delta t$, to the final value $s_{1}$ at the end of this interval. Thus, the final elastic deformation $s$ of the roughness after both analysed phases of motion, after the lapse of time $\Delta t$, is equal to:

$$
s(t+\Delta t)=s_{1}=s_{1}^{\prime}+\Delta s_{1}^{\prime} .
$$

Increment $\Delta s_{1}^{\prime}$ can be determined with the use of Eq. (3). The following dependence is obtained:

$$
\Delta s_{1}^{\prime}=\left(v_{r 2}-\frac{\left|v_{\mathrm{r} 2}\right|}{g\left(v_{\mathrm{r} 2}\right)} \cdot s_{1}^{\prime}\right) \cdot \Delta t
$$


where $v_{\mathrm{r} 2}$ is an average velocity of end point $M$ along the straight line $N_{1}^{\prime} M_{1}^{\prime \prime}$ and its direction is defined by angle $\beta$ (Fig. 1c). The value of $v_{\mathrm{r} 2}$ can be determined by the relationship analogous to $v_{\mathrm{r} 1}$, hence:

$$
v_{\mathrm{r} 2} \approx \frac{N_{1}^{\prime} M_{1}^{\prime \prime}-s_{1}^{\prime}}{\Delta t}
$$

where, based on Fig. 1c, we have:

$$
N_{1}^{\prime} M_{1}^{\prime \prime}=\sqrt{\left(s_{1}^{\prime} \cdot \cos \alpha\right)^{2}+\left(s_{1}^{\prime} \cdot \sin \alpha+\eta \cdot \Delta y_{p}\right)^{2}}
$$

After evaluation of the value $s$ and direction $\beta$ of elastic deformation of element $M N$ in the plane of sliding, at time interval $\Delta t$, and value of relative velocity $v_{\mathrm{r}}=v_{\mathrm{r} 2}$ at this moment, and knowing the values of constants $k_{\mathrm{t}}, h_{\mathrm{t}}, h_{\mathrm{v}}, v_{\mathrm{s}}, F_{\mathrm{C}}$, and $F_{\mathrm{S}}$, it is possible to determine, using relationships (2-6), an instantaneous magnitude of friction force $\vec{F}_{\mathrm{F}}$ at this instant. The components of this force along $x$ and $y$ axes can be determined according to the dependences:

$$
\begin{aligned}
& F_{\mathrm{F} x}=F_{\mathrm{F}} \cdot \cos \beta, \\
& F_{\mathrm{F} y}=F_{\mathrm{F}} \cdot \sin \beta .
\end{aligned}
$$

The coordinates $M_{1 x}$ and $M_{1 y}$ of point $M_{1}$, at which the end point $M$ of element $M N$ modelling roughness protrusion would be placed after the lapse of time interval $\Delta t$, i.e. at the beginning of the next time interval, are dependent on coordinates $x$ and $y$ of point $N_{1}^{\prime}$, and they can be defined as follows:

$$
\begin{aligned}
& M_{1 x}=M_{x}(t+\Delta t)=x-s_{1} \cdot \cos \beta, \\
& M_{1 y}=M_{y}(t+\Delta t)=y+s_{1} \cdot \sin \beta .
\end{aligned}
$$

At the succeeding time intervals, the described above cycle is repeated.

In sliding motion, the value of driving force $F_{\mathrm{d}}$ indispensable for sustaining this motion relates to the average magnitude of the component of friction force in the direction of sliding. So if the sliding is realised in the direction of $x$ axis the magnitude of the driving force can be calculated according to the following relationship:

$$
F_{\mathrm{d}}=\tilde{F}_{\mathrm{F} x}=\frac{1}{n} \sum_{i=1}^{n} F_{\mathrm{F} x_{i}}(t+i \Delta t)
$$

where $n-$ a number of time intervals $\Delta t$, to which the vibration cycle $T$ was sub-divided.

The above-described method of calculating the friction force in sliding motion, utilizing our model, at transverse ground vibrations in a single time increment $\Delta t$ can be presented in the form of the following algorithm (Fig. 2): The calculations protocol and sequence of estimations in individual blocks of the algorithm is presented in Fig. 3:

Based on the above algorithm, a computational model was developed in the Matlab/Simulink environment for analysing the influence of tangential transverse vibrations on the friction force. The correctness of analytical relationships and that of the calculation algorithm as well as of the numerical procedures developed was verified by performing appropriate tests in which the friction force profiles determined with the use of the developed model were compared with those determined according to the classical LuGre model. Figure 4a presents such a comparison for the $1 D$ model in the absence of vibrations, whilst in Fig. $4 \mathrm{~b}$ the comparison in the case when the upper body is completely immobilised-in the absolute system: $x=x(t)=0$ and $y=y(t)=0$, and the support is set in vibrating motion. In each case, the full compliance of the compared force profiles is apparent. 


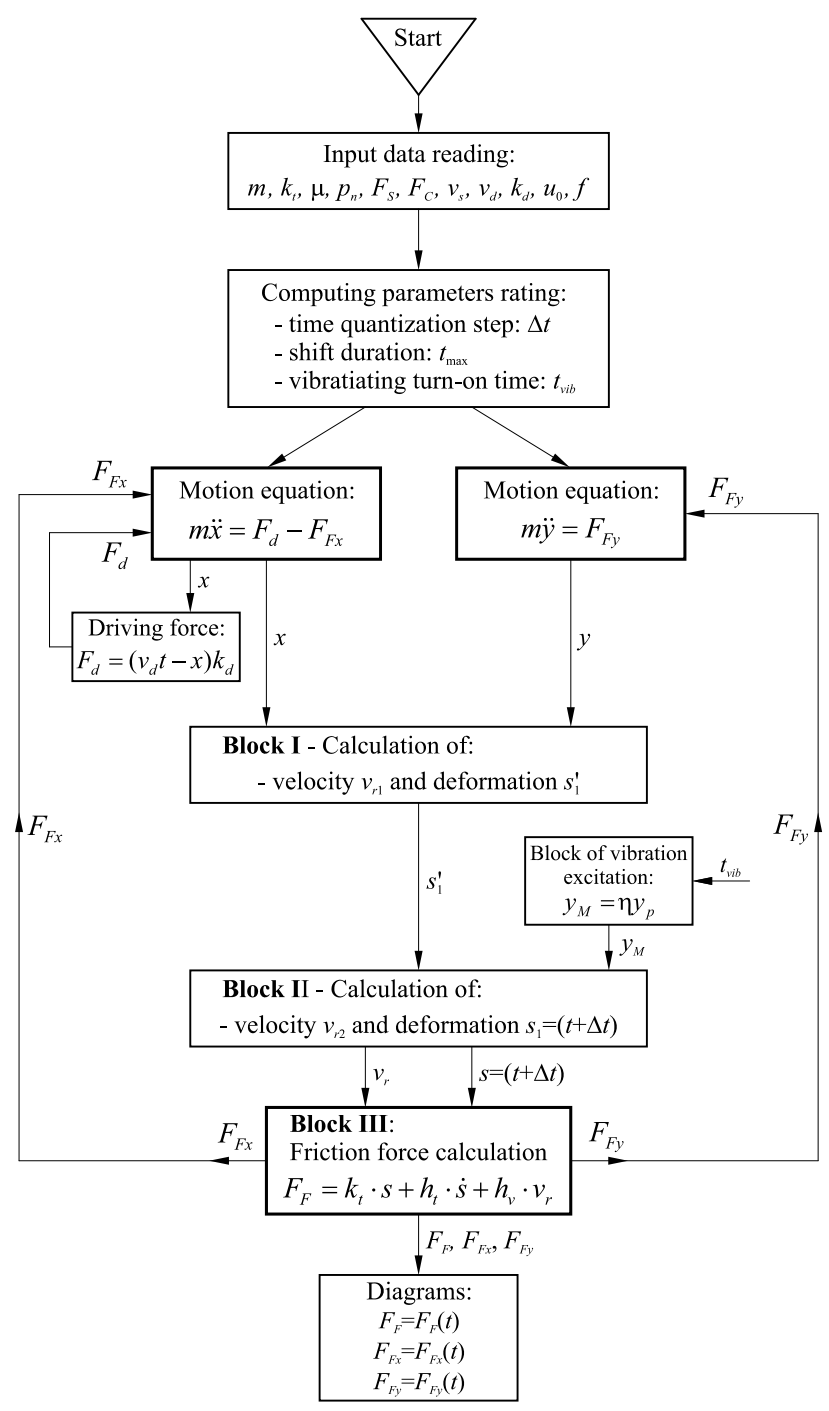

Fig. 2 Algorithm for calculating the friction force in sliding motion at transverse support vibrations

Block I - the first phase of motion

\begin{tabular}{|lc|}
\hline Calculated: & equation: \\
$\qquad(t)$ & $(1)$ \\
$M N_{1}^{\prime}$ & $(14)$ \\
$v_{r 1}$ & $(13)$ \\
$\Delta s$ & $(12)$ \\
$s_{1}^{\prime}$ & $(11)$ \\
New coordinates of point \\
$M$ after end of stage I: \\
$M_{1 x}^{\prime}$ & $(15)$ \\
$M_{1 y}^{\prime}$ & $(16)$
\end{tabular}

Block II - the second phase of motion

$$
\downarrow \begin{aligned}
& \Delta y_{M} \\
& N_{1}^{\prime} M_{1}^{\prime \prime} \\
& v_{r 2} \\
& \Delta s_{1} \\
& s(t+\Delta t)
\end{aligned}
$$$$
\text { Calculated: equation: }
$$

Coordinates of point $M$ after the lapse of $\Delta t$ :

$$
\downarrow \begin{aligned}
& M_{1 x} \\
& M_{1 y}
\end{aligned}
$$

Block III - friction force calculation

Calculated: $\quad$ equation:
\begin{tabular}{|lc}
$g\left(v_{r}\right)$ & $(4)$ \\
$h\left(v_{r}\right)$ & $(6)$ \\
$\dot{S}$ & $(3)$ \\
$F_{F}$ & $(2)$ \\
$F_{F x}$ & $(26)$ \\
$F_{F y}$ & $(27)$ \\
Results visualisation \\
Start of the next $\Delta t$
\end{tabular}

Fig. 3 The calculations protocol and sequence of estimations in individual blocks of the algorithm 

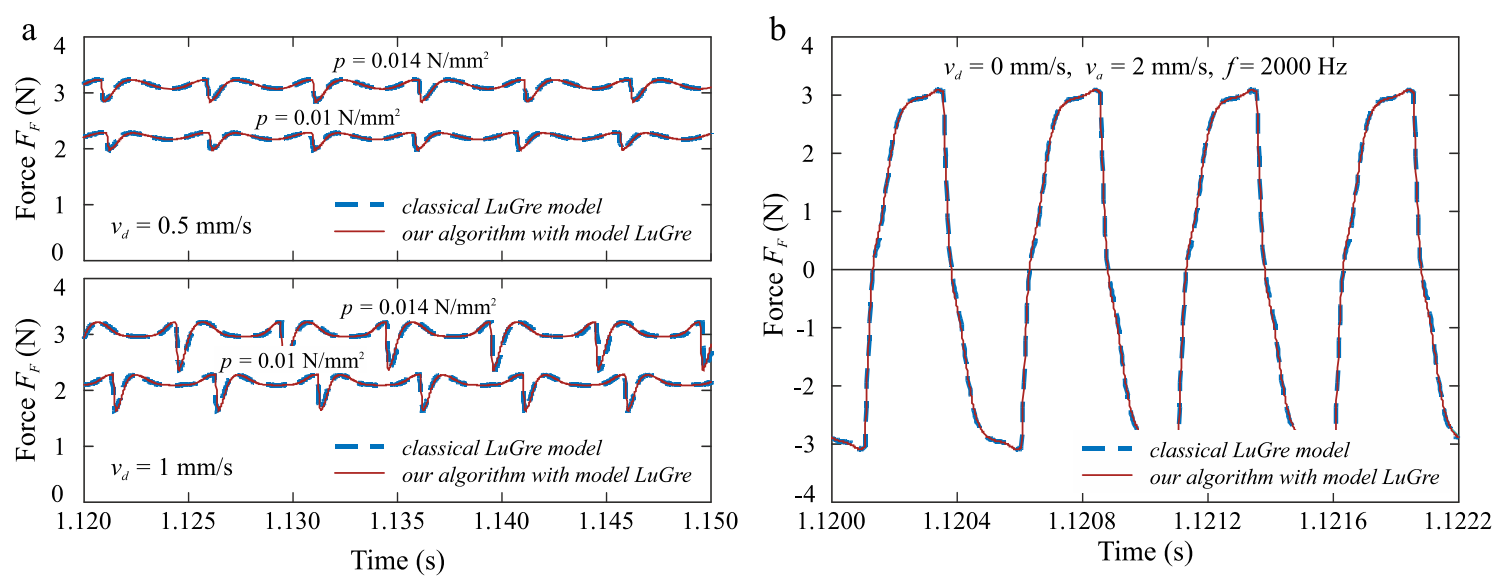

Fig. 4 Comparison of the friction force profiles according to the classical LuGre model and those according to the developed model: a $1 D$ model in the absence of vibrations, at different driving velocities; $\mathbf{b}$ the friction force profiles with the upper body completely immobilised and the support set in vibrating motion

\section{Simulating analyses}

The developed model has been used in simulating analyses. Performing these analyses required the assumption of a certain value of coefficient $\eta$ of vibrations transfer, from the support to the shifted body. The value of this coefficient resides within the boundaries of $0 \leq \eta \leq 1$. It can be determined based on experimental data by tuning in the model to the real object. The tuned-in parameter is just the value of coefficient $\eta$. The procedure is described in paper [10].

In the simulating analyses presented in this work, it has been assumed that: $\eta=0.71$, the mass of the shifted body is $m=0.56 \mathrm{~kg}$, and the normal pressures are $p_{n}=0.014 \mathrm{~N} / \mathrm{mm}^{2}$. These data are the same as in experimental tests carried out for verification of the developed model. In computations, it has been assumed also that the rigidity of the drive is $k_{\mathrm{d}}=0.92 \mathrm{~N} / \mu \mathrm{m}$, the coefficient of tangential stiffness of the contact is $k_{\mathrm{t}}$ $=62.86 \mathrm{~N} / \mu \mathrm{m}$, and the friction coefficient is $\mu=0.178$. These values were determined in experimental tests. On the basis of experiment, it was assumed moreover that $F_{\mathrm{S}}=1.1 F_{\mathrm{C}}$, and from the literature [15] it has been taken that $v_{\mathrm{e}}=v_{\mathrm{S}}=0.001 \mathrm{~mm} / \mathrm{s}$. Because the analyzed contact was dry, it has been assumed that $h_{\mathrm{v}}=0$.

Figure 5 presents the profiles of the resultant friction force $F_{\mathrm{F}}$ and its components $F_{\mathrm{F} x}$ and $F_{\mathrm{F} y}$ in sliding motion over the support excited into vibration motion in transverse direction. These profiles were generated for a variety of driving speeds $v_{\mathrm{d}}$ equal to: $0.1,0.3,0.8,2$, and $4 \mathrm{~mm} / \mathrm{s}$. The frequency of support vibrations was $f=3000 \mathrm{~Hz}$, and the amplitude of their velocity $=2 \mathrm{~mm} / \mathrm{s}$.

Figure 6, in turn, presents analogous profiles determined at vibrations frequency $f=3000 \mathrm{~Hz}$ and constant driving velocity $v_{\mathrm{d}}=0.5 \mathrm{~mm} / \mathrm{s}$, for varying amplitudes of vibrations velocity $v_{\mathrm{a}}$ equal to: $0.3,0.6,1.5$, 4 , and $12 \mathrm{~mm} / \mathrm{s}$.

It is seen from the graphs presented that transverse vibrations of the support significantly influence the profile of the friction force in sliding motion. From the practical point of view, most important is the change, i.e. reduction of the $F_{\mathrm{F} x}$ component parallel to the direction of shifting at the macro-scale, because the average value of this component is equal to the driving force $F_{\mathrm{d}}$ essential for initiating the sliding motion of the body and for maintaining it. It is seen that at a given driving velocity the friction force can be controlled by imposing vibrating motions to the support at designated frequency and amplitude. A change in the driving velocity during sliding performed over the vibrating support significantly changes the level of friction forces.

At other frequencies, the influence of support vibrations on the driving force and friction force is qualitatively similar to that described above. As an example, Fig. 7 presents profiles of the friction force and its components in sliding motion over the support vibrating in the direction perpendicular to that of the shifting direction at the frequency $f=2000 \mathrm{~Hz}$, amplitude of vibrations velocity $v_{\mathrm{a}}=2.0 \mathrm{~mm} / \mathrm{s}$, and varying drive velocities $v_{\mathrm{d}}$.

Comparing the respective profiles of friction force $F_{\mathrm{F}}$ and its components $F_{\mathrm{F} x}$ and $F_{\mathrm{F} y}$ in sliding motion over a vibrating support, generated for frequency $f=3000 \mathrm{~Hz}$ (Fig. 4) with the profiles related to frequency $f=2000 \mathrm{~Hz}$ (Fig. 7), it is seen that at the same vibrations amplitude such, rather considerable, change of 

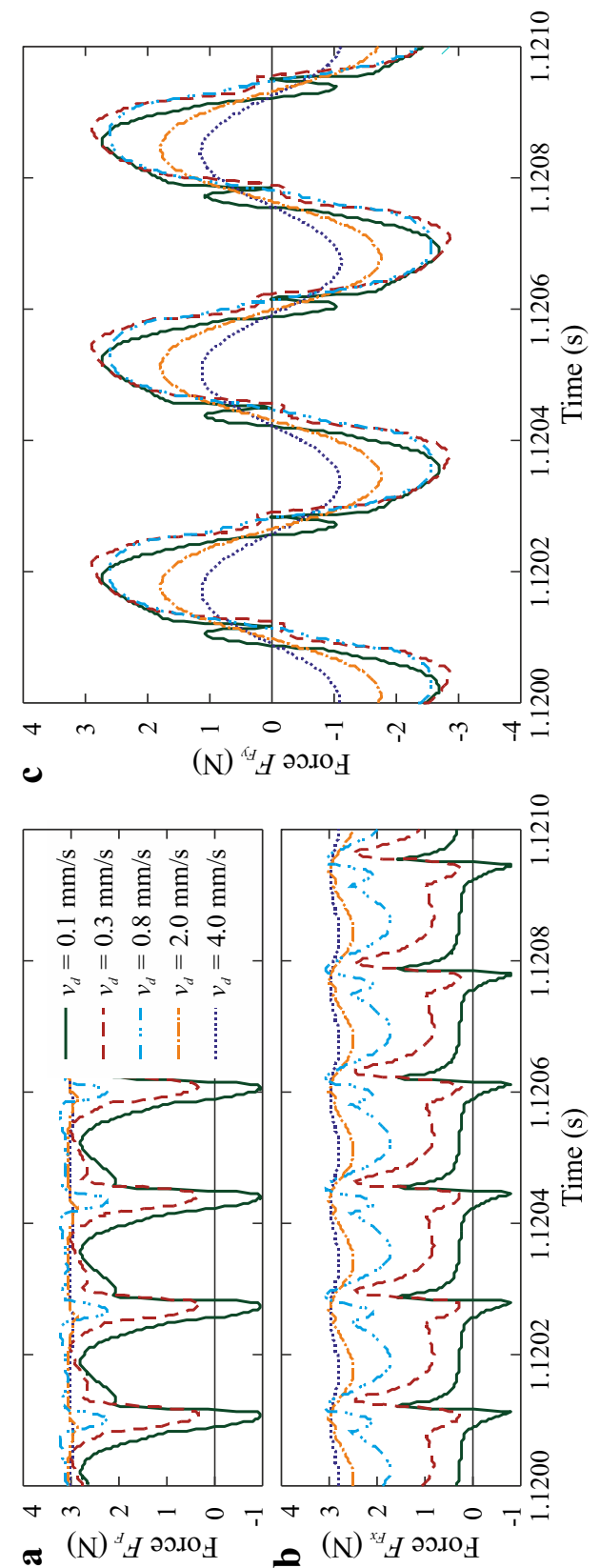

0
$\vdots$
0
11
2
2
01
0
8
0
11
4
0
2
2
20
0
0
0
0

$\stackrel{\infty}{:}$

$\stackrel{8}{\Xi}$

$\stackrel{8}{9}$

莺

.

迹

ส

这

苞

है

$\stackrel{\mathscr{0}}{\mathscr{0}}$

ส

连

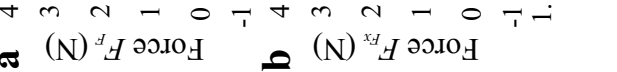

.0ำ

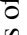

$\frac{0}{0}$ 

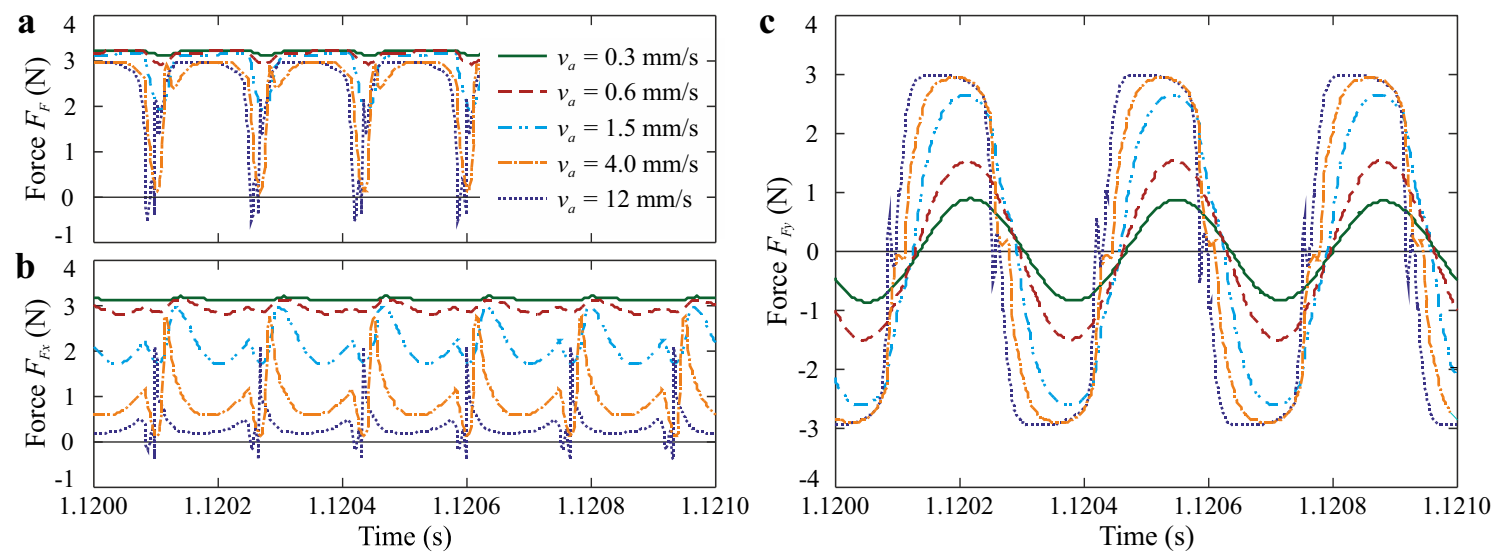

Fig. 6 Profiles of friction force $F_{\mathrm{F}}$ and its components $F_{\mathrm{Fx}}$ and $F_{\mathrm{Fy}}$ in relation to the amplitude of vibrations velocity $v_{\mathrm{a}} ; f=$ $3000 \mathrm{~Hz}, v_{\mathrm{d}}=0.5 \mathrm{~mm} / \mathrm{s}$
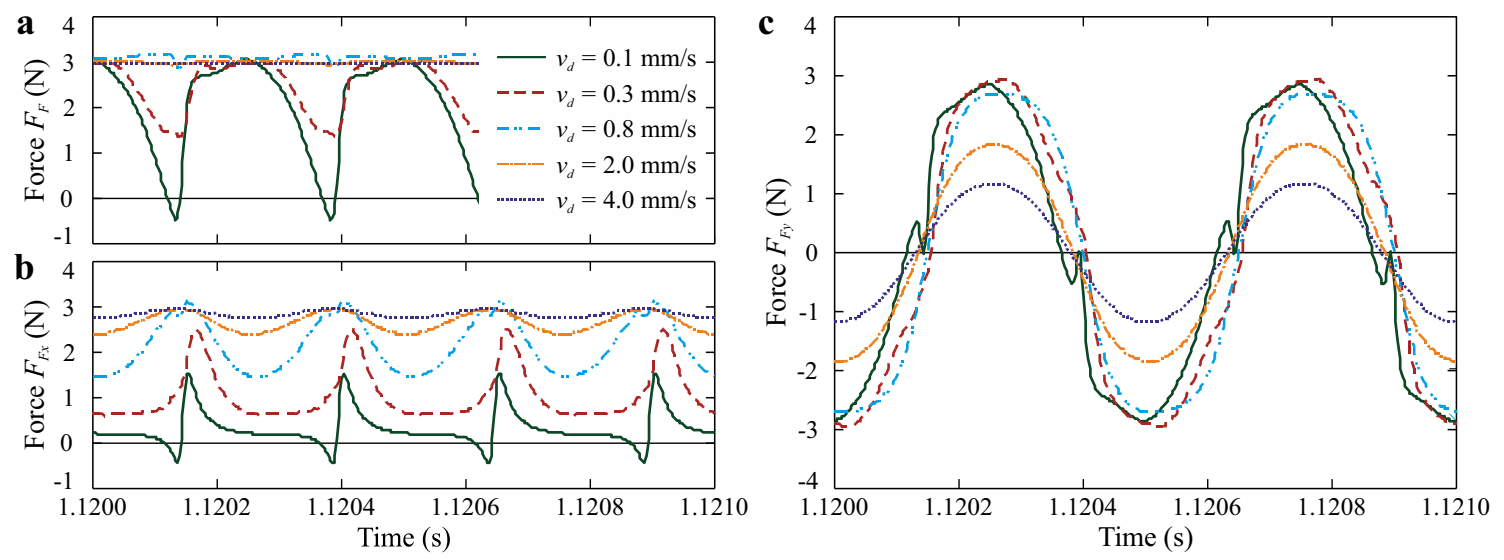

Fig. 7 Profiles of friction force $F_{\mathrm{F}}$ and its components $F_{\mathrm{Fx}}$ and $F_{\mathrm{Fy}}$ in relation to the driving speed $v_{\mathrm{d}} ; f=2000 \mathrm{~Hz}$ and $v_{\mathrm{a}}=$ $2 \mathrm{~mm}$

vibration frequency of support did not influence significantly the time profiles of friction force $F_{\mathrm{F}}$ and its components $F_{\mathrm{F} x}$ and $F_{\mathrm{F} y}$. Changed are the average values of these components.

In Fig. 8, collective graphs are presented regarding the variability of an average friction force $\tilde{F}_{F x}$ in sliding direction over a vibrating support, which correspond to driving force $F_{\mathrm{d}}$ essential for the initiation of sliding motion of the body and maintaining it. They were determined according to Eq. (30). The graphs were generated at vibration frequency $f=3000 \mathrm{~Hz}$, for various driving velocities $v_{\mathrm{d}}$, and for various amplitudes $v_{\mathrm{a}}$ of vibration velocities. It can be seen how the value of $\tilde{F}_{F x}$ is reduced at constant frequency of vibration with increasing the amplitude of vibration velocity and decreasing the driving velocity.

\section{The experimental verification of the model}

Verification of the developed model was carried out by comparison of the results of simulation analyses performed with the use of this model with corresponding results of experiments utilising a dedicated experimental rig described in detail in papers $[10,14]$. Experimental tests were carried out on a frictional pair made of steel. The roughness of contact surfaces defined by parameter $R_{a}$ was $R_{a} / R_{a}=0.26 / 0.61 \mu \mathrm{m} / \mu \mathrm{m}$.

Comparative analyses were performed in the frequency band $f$ of $1000 \div 4000 \mathrm{~Hz}$ and in the range of driving velocity from $v_{\mathrm{d}}=0.1$ to $v_{\mathrm{d}}=5 \mathrm{~mm} / \mathrm{s}$. The range of normal pressure was from $p_{\mathrm{n}}=0.014$ to $p_{\mathrm{n}}=$ $0.07 \mathrm{~N} / \mathrm{mm}^{2}$. In computations, the same values of parameters $m, k_{\mathrm{d}}, k_{\mathrm{t}}, \mu, v_{\mathrm{s}}, h_{\mathrm{v}}$, and $F_{\mathrm{S}}$ were taken as assumed in the formerly presented examples. The amplitude $v_{\mathrm{a}}$ of the vibration velocity was a variable parameter. 

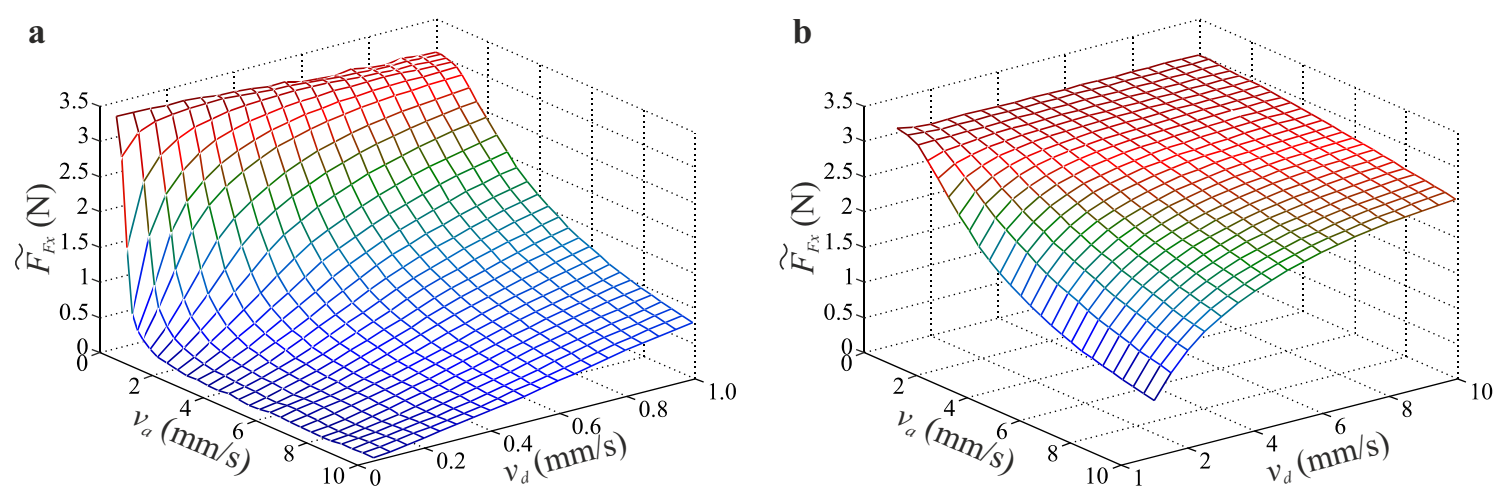

Fig. 8 Profiles of average friction force $\tilde{F}_{\mathrm{F} x}$ variability in the sliding direction as a function of driving velocity $v_{\mathrm{d}}$ and amplitude $v_{\mathrm{a}}$ of vibration velocity; a for $v_{\mathrm{d}} \leq 1, \mathbf{b}$ for $1 \leq v_{\mathrm{d}} \leq 10$
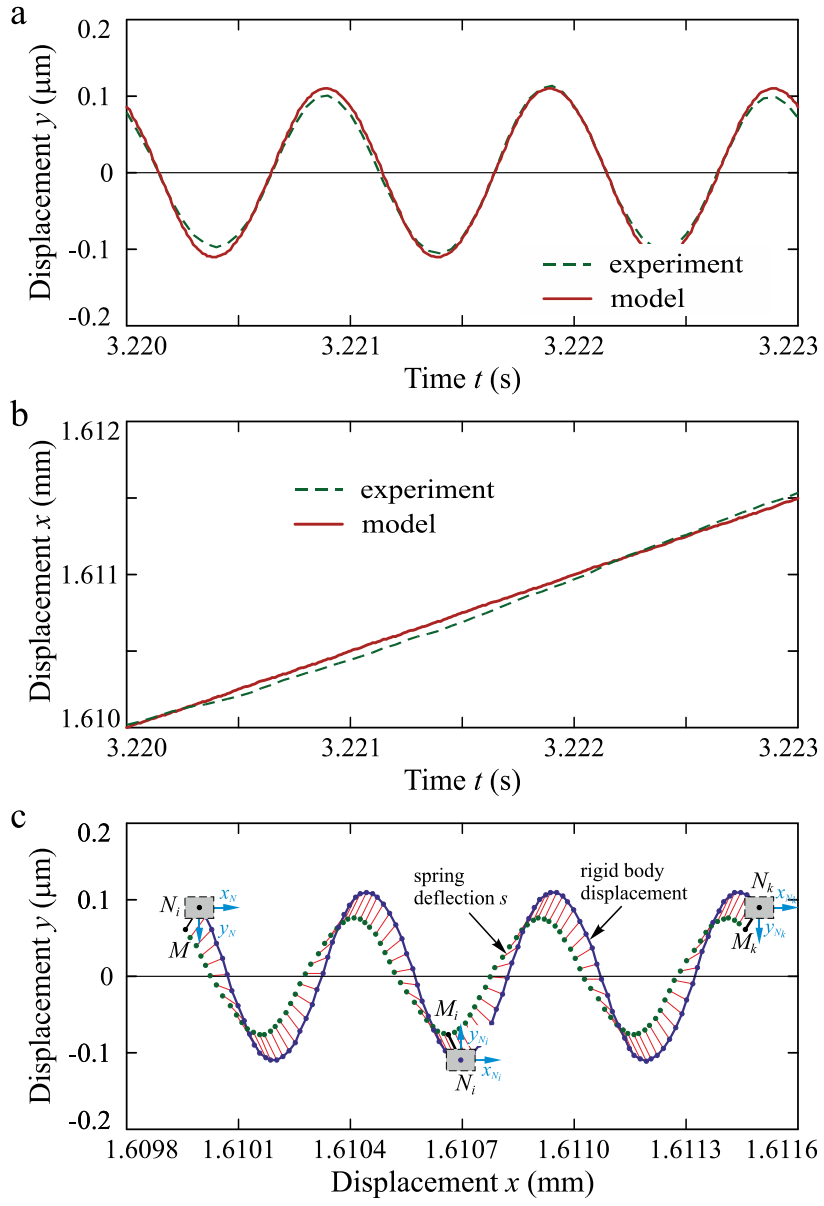

Fig. 9 a and $\mathbf{b}$ time profiles $x(t)$ and $y(t)$ of displacements of the shifted body; c trajectories of movement of the ends $M$ and $N$ of the element modeling the contact zone; $v_{\mathrm{d}}=0.5 \mathrm{~mm} / \mathrm{s}, f=1000 \mathrm{~Hz}$ and $\mu_{0}=0.1512 \mu \mathrm{m}$

At the preliminary stage, the time profiles $x(t)$ and $y(t)$ of displacements of the shifting body determined experimentally, and those generated numerically with the use of the developed model, were compared. An example of comparison of such profiles for two sets of input data is shown in Figs. 9 and 10.

The first one presents the comparison at the driving velocity $v_{\mathrm{d}}=0.5 \mathrm{~mm} / \mathrm{s}$ with the parameters of forced support vibrations: $f=1000 \mathrm{~Hz}$ and $u_{o}=0.1512 \mu \mathrm{m}$, whilst the second with the same driving velocity and the identical frequency $f$ but at an increased vibrations amplitude $u_{o}=0.3676 \mu \mathrm{m}$. Good agreement of the 

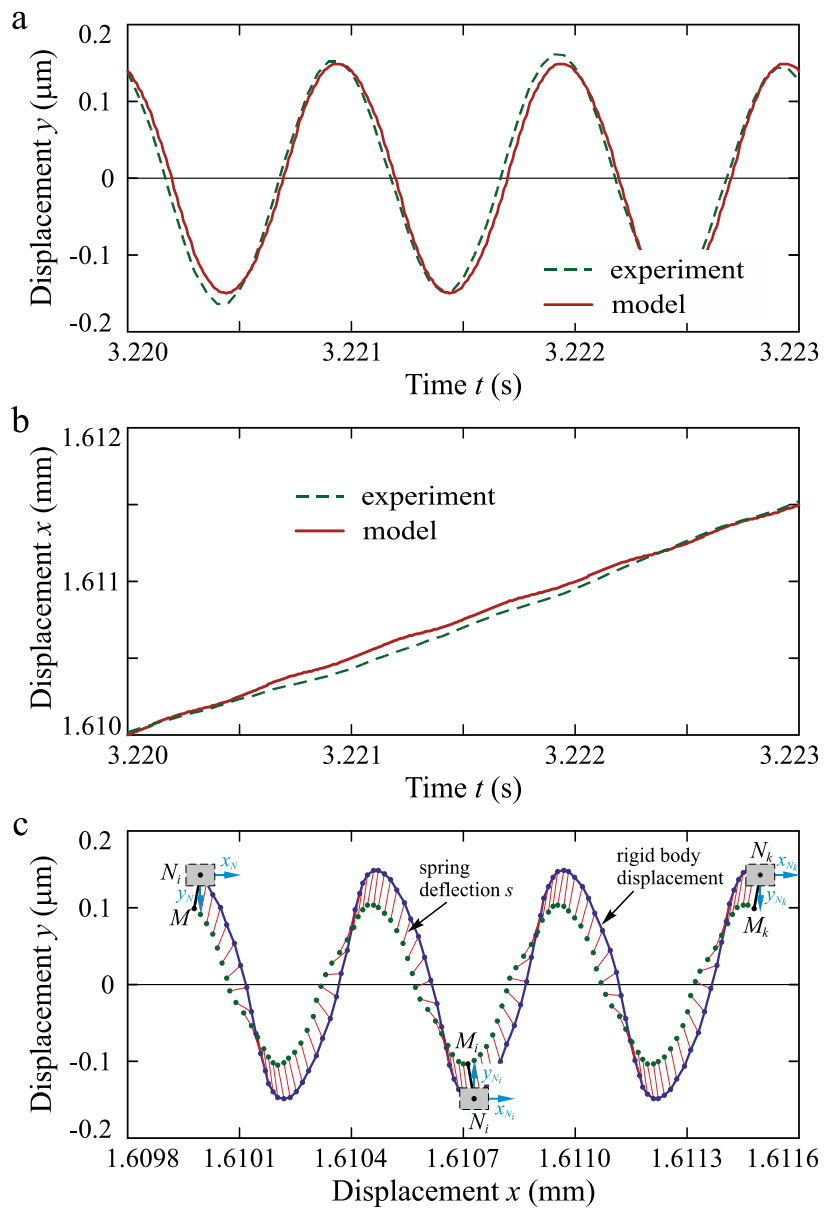

Fig. 10 a and b time profiles $x(t)$ and $y(t)$ of displacements of the shifted body; c trajectories of movement of the ends $M$ and $N$ of the element modeling the contact zone; $v_{\mathrm{d}}=0.5 \mathrm{~mm} / \mathrm{s}, f=1000 \mathrm{~Hz}$, and $\mu_{0}=0.3676 \mu \mathrm{m}$

compared profiles is seen. Additionally, these Figures (Figs. 9c and 10c) show the trajectories of motion of the shifted body (point $N$ ) and the $M$-end of the element $M N$ modeling the deformable contact zone, as determined by numerical analyses.

Further verification was carried out in two stages. At the first of these, the average friction force in the direction of motion generated by the numerical computations performed with the use of the developed model was compared with corresponding experimental results. At the second one, such comparison is carried out for the time profiles of friction force $F_{\mathrm{F}}$ and its components $F_{\mathrm{F} x}$ and $F_{\mathrm{F} y}$. The examples of obtained results for average friction force reduction are presented in Fig. 11, and for time profiles of friction force $F_{\mathrm{F}}$ and its components $F_{\mathrm{F} x}$ and $F_{\mathrm{F} y}$ in Fig. 12.

A good compliance of results from simulating analyses with experimental ones can be seen. This is a testimony to the correctness of the developed model.

Figure 12, however, shows a certain mismatch between the time profiles of the resultant friction force $F_{\mathrm{F}}$ and its component $F_{\mathrm{F} x}$ estimated using the model and that determined experimentally, whilst showing good compliance of respective profiles of the $F_{\mathrm{F} y}$ component. The Figure shows that the profiles determined experimentally are smoother. This may be due to a limited sensitivity of the measuring sensors and limited sampling frequency of the recorded signals arising from inherent capability of the measuring card used in the experiments.

It should be, however, emphasised that the average values of the resultant friction force and its two components estimated from the model and determined experimentally differ very slightly, which can be seen from the dataset presented in Table 1: 

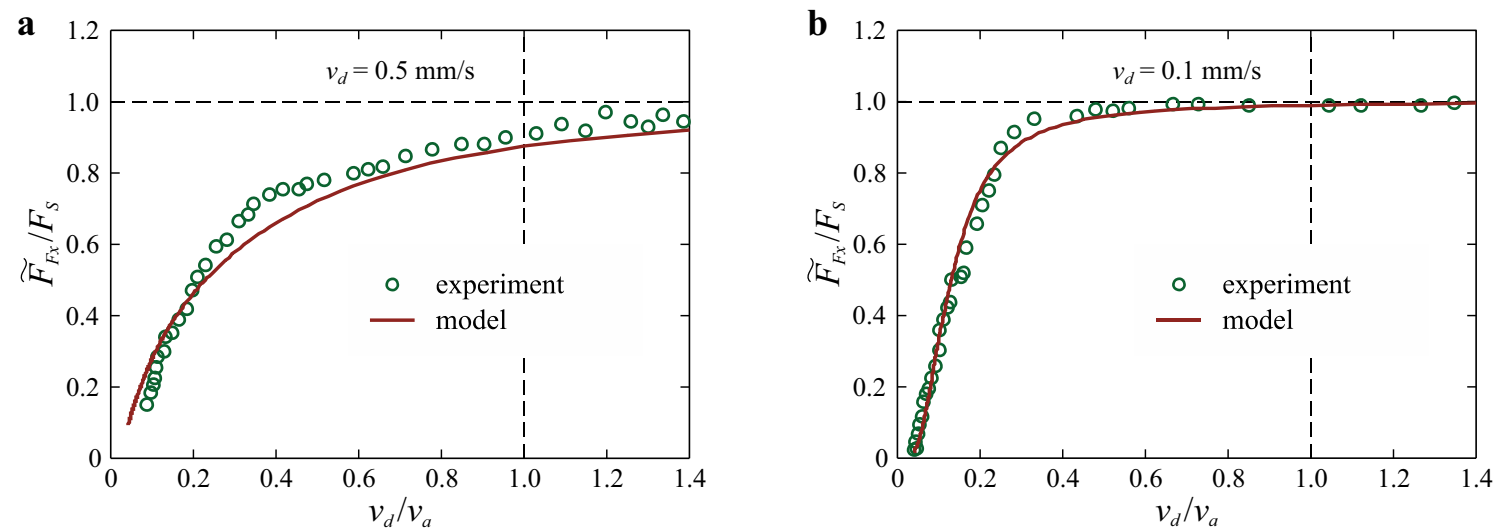

Fig. 11 Comparison of simulating analyses results with those determined experimentally; $\mathbf{a} f=1000 \mathrm{~Hz}, p_{\mathrm{n}}=0.014 \mathrm{~N} / \mathrm{mm}^{2}, \mathbf{b} f=$ $3000 \mathrm{~Hz}, p_{\mathrm{n}}=0.021 \mathrm{~N} / \mathrm{mm}^{2} ; k_{\mathrm{d}}=0.92 \mathrm{~N} / \mu \mathrm{m}, k_{\mathrm{t}}=62,86 \mathrm{~N} / \mu \mathrm{m}, \mu=0.178$
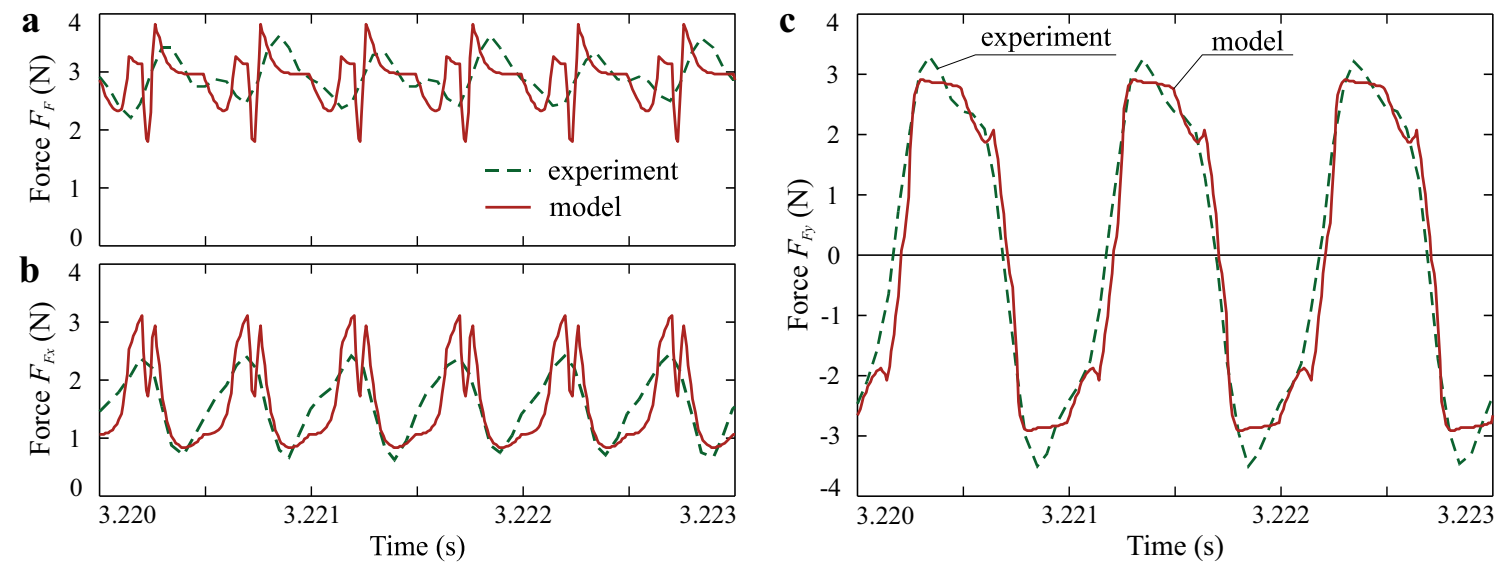

Fig. 12 Comparison of friction force profiles and its components determined experimentally and with the use of developed model; $\mathbf{a}$ friction force $F_{\mathrm{F}}, \mathbf{b}$ component $F_{\mathrm{F} x}, \mathbf{c}$ component $F_{\mathrm{F} y} ; f=1000 \mathrm{~Hz}, v_{\mathrm{d}}=0.5 \mathrm{~mm} / \mathrm{s}, v_{\mathrm{a}}=2.31 \mathrm{~mm} / \mathrm{s}$

Table 1 Average values of the friction force and its components

\begin{tabular}{lrr}
\hline & Model & Experimental \\
\hline$F_{\mathrm{F}},[\mathrm{N}]$ & 2.8629 & 2.9391 \\
$F_{\mathrm{F} x},[\mathrm{~N}]$ & 1.5469 & 1.5899 \\
$F_{\mathrm{F} y},[\mathrm{~N}]$ & 0.0086 & -0.0652 \\
\hline
\end{tabular}

\section{Summary and conclusions}

A novel computational model presented in this work very well represents changes in friction forces taking place within real frictional couplings under the influence of transverse tangential vibrations of the elastic support. Hence, it can be utilised in simulating analyses and also in real engineering systems for controlling the friction force by such vibrations.

In contrast to the models presented by other authors this model considers the mass of the shifted, and subjected to vibration, body. Hence, it is a dynamic model. Also, unlike other models, the presented model assumes that vibrations are not imposed directly on the shifting body but are transferred to it from the vibrating elastic support. This is a variant considerably more difficult to analyse due to the fact that vibrations of the support are not transferred to the shifted body in their entirety.

Open Access This article is licensed under a Creative Commons Attribution 4.0 International License, which permits use, sharing, adaptation, distribution and reproduction in any medium or format, as long as you give appropriate credit to the original author(s) and the source, provide a link to the Creative Commons licence, and indicate if changes were made. The images or other 
third party material in this article are included in the article's Creative Commons licence, unless indicated otherwise in a credit line to the material. If material is not included in the article's Creative Commons licence and your intended use is not permitted by statutory regulation or exceeds the permitted use, you will need to obtain permission directly from the copyright holder. To view a copy of this licence, visit http://creativecommons.org/licenses/by/4.0/.

\section{References}

1. Matunaga, S., Onoda, J.: New gravity compensation method by dither for low-g simulation. J. Spacecr. Rockets 32(2), 364-369 (1995)

2. Storck, H., Littmann, W., Wallaschek, J., Mracek, M.: The effect of friction reduction in presence of ultrasonic vibrations and its relevance to travelling wave ultrasonic motors. Ultrasonics 40, 379-383 (2002)

3. Kumar, V.C., Hutchings, I.M.: Reduction of the sliding friction of metals by the application of longitudinal or transverse ultrasonic vibration. Tribol. Int. 37, 833-840 (2004)

4. Tsai, C.C., Tseng, C.H.: The effect of friction reduction in the presence of in-plane vibrations. Arch. Appl. Mech. 75, 164-176 (2006)

5. Popov, V.L., Starcevic, J., Filipov, A.E.: Influence of ultrasonic in-plane oscillations on static and sliding friction and intrinsic length scale of dry friction processes. Tribol. Lett. 39, 25-30 (2010)

6. Qu, J.J., Zhou, N.N., Wang, J.L.: Experimental study of air squeeze effect on high-frequency friction contact. Tribol. Int. 43, 2190-2195 (2010)

7. Teidelt, E., Starcevic, J., Popov, V.L.: Influence of ultrasonic oscillation on static and sliding friction. Tribol. Lett. 49, 51-62 (2012)

8. Gutowski, P., Leus, M.: The comparative analyses of the influence of tangential transverse and tangential longitudinal vibrations on friction force in sliding motion. Tribologia 2, 69-79 (2013). (in Polish)

9. Wang, P., Ni, H., Wang, R., Li, Z., Wang, Y.: Experimental investigation of the effect of in-plane vibrations on friction for different materials. Tribol. Int. 99, 237-247 (2016)

10. Gutowski, P., Leus, M.: Computational model for friction force estimation in sliding motion at transverse tangential vibrations of elastic support. Tribol. Int. 90, 455-462 (2015)

11. Dahl, P.R.: Solid friction model. Technical Report TOR - 0158H3107-181-1. The Aerospace Corporation, El Segundo, CA (1968)

12. de Canudas, W.C., Olsson, H., Aström, K.J., Lischinsky, P.: A new model for control of systems with friction. IEEE Trans. Autom. Control 40(3), 419-425 (1995)

13. Olsson, H., Aström, K.J., de Canudas, W.C., Gäfvert, M., Lischinsky, P.: Friction models and friction compensation. Eur. J. Control 4, 175-195 (1998)

14. Leus, M., Gutowski, P.: Experimental and simulation analyses of the influence of tangential transverse vibrations on friction force in sliding motion. Modelowanie Inżynierskie 16(47), 129-133 (2013). (in Polish)

15. Olsson, H.: Control systems with friction. Doctoral thesis. Department of Automatic Control, Lund Institute of Technology (1996)

Publisher's Note Springer Nature remains neutral with regard to jurisdictional claims in published maps and institutional affiliations. 